\title{
Metabolic profiling of liver and faeces in mice infected with echinococcosis
}

\author{
Mingxing Zhu ${ }^{1,2,3+}$, Xiancai Du ${ }^{2,3 \dagger}$, Hongxia Xu ${ }^{1,2+}$, Songhao Yang ${ }^{2,3}$, Chan Wang ${ }^{2,3}$, Yazhou Zhu ${ }^{2,3}$,
}

Tingrui Zhang ${ }^{2,3}$ and Wei Zhao ${ }^{1,2^{*}}$

\begin{abstract}
Background: Echinococcosis is a severe zoonotic parasitic disease which severely affects the health of the hosts. The diagnosis of echinococcosis depends mainly on imaging examination. However, the patient is often in the late stage of the disease when the symptoms appear, thus limiting the early diagnosis of echinococcosis. The treatment and prognosis of the patients are hampered because of long-term asymptomatic latency. Metabolomics is a new discipline developed in the late 1990s. It reflects a series of biological responses in pathophysiological processes by demonstrating the changes in metabolism under the influence of internal and external factors. When the organism is invaded by pathogens, the alteration in the characteristics of metabolites in cells becomes extremely sensitive. Here, we used a metabolomics approach involving liquid chromatography coupled with tandem mass spectrometry (LCMS/MS) to determine the molecular mechanism of cystic echinococcosis (CE) and to develop an effective method for CE diagnosis.

Methods: Twenty 8-week-old female BALB/c mice were divided into normal and Echinococcus granulosus infection groups. To develop the E. granulosus infection model, mice were infected with protoscoleces. Six weeks later, the abdomens of the mice showed significant bulging. An LC-MS/MS system-based metabolomics approach was used to analyse the liver and faeces to reveal the metabolic profiles of mice with echinococcosis.

Results: We found that the metabolism of nucleotides, alkaloids, amino acids, amides, and organic acids in mice is closely interrelated with E. granulosus infection. In the liver, the metabolic pathways of tyrosine and tryptophan biosynthesis; phenylalanine, valine, leucine and isoleucine biosynthesis; and phenylalanine metabolism were notably associated with the occurrence and development of hydatid disease, and in the faeces, pantothenate and CoA biosynthesis are thought to be closely associated with the development of CE.
\end{abstract}

Conclusion: The metabolomics approach used in this study provides a reference for a highly sensitive and specific diagnostic and screening method for echinococcosis.

Keywords: Echinococcosis, Metabolomics, Metabolites, Liquid chromatography coupled with tandem mass spectrometry (LC-MS/MS)

*Correspondence: Weizhao@nxmu.edu.cn

${ }^{\dagger}$ Mingxing Zhu, Xiancai Du and Hongxia Xu contributed equally to this work

${ }^{1}$ Center of Scientific Technology of Ningxia Medical University,

Yinchuan 750004, Ningxia Hui Autonomous Region, People's Republic of China

Full list of author information is available at the end of the article

\section{Background}

Cystic echinococcosis (CE) or hydatidosis is a type of near-cosmopolitan zoonosis caused by Echinococcus granulosus sensu lato $[1,2]$. The life cycle of E. granulosus involves two mammalian hosts. For adult tapeworms, carnivores (canines and cats) are the definitive hosts, while ungulates and rodents are intermediate hosts [3]. CE is prevalent in Western China, South America, 
Central Asia, the Mediterranean, and East Africa [4]. The main risk factors are close contact with dogs and livestock [5-8]. Humans are not usually directly involved in the spread of CE. However, humans or intermediate hosts accidentally ingest eggs, which are then incubated in the intestines and release oncospheres [9]. The oncospheres are transported to the liver through the portal vein and lymphatic vessels where they settle and develop into larvae (hydatid cysts), partly reaching the lungs and rarely reaching the brains, bones, or any other organ of a human or intermediate host $[2,10]$. The growth of CE cysts is very slow [11]. There are no significant symptoms in the early stage of CE, with more than half of cysts expressing no change in size in 10-15 years [12]. Clinical symptoms such as epigastric discomfort or loss of appetite commonly appear when the cyst becomes more than $10 \mathrm{~cm}$ long, and gradually develop, causing damage and dysfunction in the parasitized organs (mainly the liver) $[13,14]$. Clinically, patients with $\mathrm{CE}$ are in the late stage of echinococcosis when they present to the hospital. The cyst is frequently misdiagnosed as a tumour [15]. Therefore, determination of a positive and effective approach to discriminate patients with $\mathrm{CE}$ from healthy individuals is important for the early diagnosis and treatment of echinococcosis, which can significantly improve the survival rate of patients.

Metabolomics, a new high-throughput sequencing technology introduced in recent years, is an important branch of omics and has great potential in drug toxicity or safety evaluation [16]. Metabolomics reflects many factors, such as gene changes, nutritional status, pathogenesis, natural environmental changes, drug treatment, physiological response, and pathological characteristics [17-21]. It is a dynamic observation of the occurrence and development of diseases and the transformation process [22]. In metabolomic studies, small molecule metabolites are used as the research object. It can be used to study drug metabolism, find differential metabolites, and explore the target and mechanism of action with the corresponding statistical analysis software using highly sensitive instruments (gas chromatography-mass spectrometry (GC-MS), nuclear magnetic resonance (NMR), and liquid chromatography-mass spectrometry (LC-MS/MS) [23-25]. Metabolomics has been widely used in a variety of hepatopathies to identify potential early biomarkers and metabolic pathways [26], which is a feasible measure for illustrating host-parasite interactions [27-29]. The metabolic state and biochemical activity of cells or tissues can be directly reflected by metabolites such as amino acids, lipids, or sugars [30,31]. Metabolic profiling approaches have been widely used in various studies on diseases caused by flatworms such as Fasciola hepatica and roundworms such as Onchocerca volvulus [32-35]. Nevertheless, reports on the application of the LC-MS/MS-based metabolomics approach in echinococcosis are limited [36]. In this study, we used a LC-MS/MS system-based metabolomics approach and multivariate statistical analyses to investigate the molecular mechanism of $\mathrm{CE}$ and to provide a potential valuable reference for the diagnosis of $\mathrm{CE}$.

\section{Methods}

\section{Establishment of hydatidosis model}

Twenty 8-week-old female BALB/c mice weighing about 18-22 g were randomly divided into two groups: normal control group $(n=10)$ and E. granulosus infection group $(n=10)$. The mice were intraperitoneally injected with protoscoleces diluted with $2 \mathrm{~mL}$ phosphate-buffered saline (PBS) (containing 2000 protoscoleces) to develop the E. granulosus infection model. The normal control group was intraperitoneally injected with the same $2 \mathrm{~mL}$ PBS. The liver and faeces of both groups were obtained by dissection after 6 weeks of feeding. The mice models infected with E. granulosus were confirmed through routine pathological section staining.

The animal experiments were implemented in strict accordance with the guidelines for laboratory animal management, with the approval of the Ningxia Medical University animal ethics committee, and in accordance with the National Institutes of Health guidelines for the care and use of laboratory animals.

\section{Sample preparation}

First, $25 \mathrm{mg}$ of hepatic and faecal samples were weighed and transferred to a new clean EP tube. Then, $500 \mu \mathrm{L}$ extract solution (acetonitrile: methanol: water $=2: 2: 1$ ) with isotopically labelled internal standard mixture was added to the samples. After $30 \mathrm{~s}$ of gentle vortexing, the samples were homogenized at $35 \mathrm{~Hz}$ for $4 \mathrm{~min}$ followed by ultrasonication in an ice-water bath for $5 \mathrm{~min}$. The homogenization and ultrasonication cycle was carried out three times. Then, the samples were incubated at $-40{ }^{\circ} \mathrm{C}$ for $1 \mathrm{~h}$ and centrifuged at $4{ }^{\circ} \mathrm{C}$ for $15 \mathrm{~min}$ at $12,000 \mathrm{rpm}$. The resulting supernatant was transferred to a fresh and clean glass bottle for analysis. A quality control sample was prepared by mixing the supernatants of all samples of liver or faeces aliquoted in equal amounts.

\section{LC-MS/MS analysis}

LC-MS/MS analyses were performed using a high-performance liquid chromatography (HPLC) system (Vanquish, Thermo Fisher Scientific, Waltham, MA, USA and Bruker BioSpin, Karlsruhe, Germany). The injection volume of the liver and faeces was $3 \mu \mathrm{L}$.

The LC-MS/MS spectra were collected by a Q Exactive HF-X Orbitrap (QE HFX) mass spectrometer in the 
control of the acquisition software (Xcalibur, Thermo Fisher Scientific). The acquisition software continuously evaluates the full scan MS spectrum in this mode. The conditions of electrospray ionization were set as follows: auxiliary gas flow rate $10 \mathrm{Arb}$, capillary temperature $320{ }^{\circ} \mathrm{C}$, sheath gas flow rate 50 Arb, full MS resolution 60,000 , collision energy 10/30/60 in NCE mode, LC-MS/ MS resolution 7500, and spray voltage $3.5 \mathrm{kV}$ (positive) or $-3.2 \mathrm{kV}$ (negative).

\section{Metabolomics statistical analysis}

The original data were converted to mzXML format using ProteoWizard (ProteoWizard, Palo Alto, CA, USA), and processed by an in-house program which was based on XCMS and was developed using R ( $\mathrm{R}$ Foundation for Statistical Computing, Vienna, Austria). Metabolites were annotated using an in-house MS2 database (BiotreeDB). The cutoff value for annotation was set to 0.3 .

\section{OPLS-DA analysis}

The data were log-transformed and UV-formatted using SIMCA software (V16.0.2, Sartorius Stedim Data Analytics $A B$, Umea, Sweden), and the first principal component was analysed by OPLS-DA modeling. The quality of the model was tested using sevenfold cross-validation.

\section{Statistical analysis}

SPSS software (version 20.0 for Windows; IBM Corporation, Armonk, NY, USA) was used to analyse the data, which have been expressed as mean $\pm \mathrm{SD}$. In all cases, $p<0.05$ was considered to be significant.

\section{Results}

\section{Multivariate statistical analysis}

To determine the metabolic changes in the liver and faeces due to echinococcosis initiated following E. granulosus infection, principal component analysis (PCA) was conducted for two groups of samples. The metabolites of the liver were found to be in moderate groupings between the infection and normal control groups under positive ion mode (POS) and negative ion mode (NEG) according to PCA (Fig. 1A, B). The interpretation rates of the first principal component (PC1) and second principal component (PC2) under POS were $33.5 \%$ and $17.6 \%$, respectively, while under NEG, the respective interpretation rates were $30.9 \%$ and $15.6 \%$. To better highlight the metabolic changes between the two groups, orthogonal projections to latent structures-discriminant analysis (OPLS-DA) was performed. The difference between the two groups was evident in the OPLS-DA score plots (Fig. 1C, D). A similar tendency was observed in the metabolic changes in faeces (Additional file 1: Fig. S1A-D).
The group separation revealed that hydatid disease could cause notable metabolic changes in the liver and faeces of mice. All the samples were within the $95 \%$ confidence interval. In addition, the OPLS-DA models were supposed to explain the predictive powers $\mathrm{Q}^{2}$ and variations $\mathrm{R}^{2} \mathrm{Y}$ with permutation tests (Fig. 1E, F, Additional file 1: Fig. S1E, F).

\section{Screening of differential metabolites}

To distinguish metabolic markers between the infection and control groups, the data are presented in the form of volcano plots. The results of the metabolic changes were determined based on variable importance in the projection $(\mathrm{VIP})>1$ and $p<0.05$. The metabolites with enormous changes have been represented with blue or red dots and larger circle shapes, located in the upper left or right corner of the volcano plots. From the metabolites of the liver, 248 and 131 distinct metabolic molecules were screened under POS and NEG (Fig. 2A, B). In the faeces, 201 and 58 various metabolites were screened under POS and NEG (Additional file 2: Fig. S2A, B). The top 15 metabolites with the most significantly different metabolism under POS and NEG in the liver and faeces are shown in Tables 1 and 2 and Additional file 3: Tables S1 and S2. In the livers from the infection group, representative substances such as bile acid (deoxyviolaceinic acid), glycerides (2-O-(alpha- $d$-mannosyl)- $d$-glycerate), amino acids (glutaminylhistidine), and nucleotides (cytidine 2,3'-cyclic phosphate) were significantly upregulated, while nucleotides (inosinic acid and 5-fluorodeoxyuridine monophosphate) were markedly downregulated under POS and in NEG. Furthermore, in the faeces of the mice with echinococcosis, metabolites such as nucleotides (dTMP), alkaloids (piperine), amino acids ( $d$-pantethine, $d$-aspartic acid, and gamma-glutamylleucine), and amides ( $N$-acetylhistamine) were upregulated, while nucleosides (5'-methylthioadenosine), amino acids (3-methylcrotonylglycine, selenocystemic acid), organic acids (hexadecanedioic acid, dimethylmalonic acid, and ascorbic acid), and alkaloids (cytokinin b) were downregulated under POS and NEG (Tables 1, 2 and Additional file 3: Tables S1 and S2). The results suggest that the metabolism of nucleotides, alkaloids, amino acids, amides, and organic acids in mice is closely interrelated with E. granulosus infection.

\section{Hierarchical clustering analysis of different metabolites}

For every sample, we calculated the Euclidean distance matrix to determine the quantitative value of the differential metabolites. The differential metabolites of the liver were clustered through the complete linkage 
Fig. 1 PCA (A, B), OPLS-DA (C, D) score plots and OPLS-DA permutation plots $(\mathbf{E}, \mathbf{F})$ in the liver. A, B PCA score plots, the abscissa $P C 1$ and the ordinate $P C 2$ represent the scores of the principal components ranking the first and the second, respectively, and different shapes of the scattered points represent the different groups of the samples. C, D OPLS-DA score plots, the ordinate $t[1] \mathrm{O}$ represents the orthogonal principal component score, the abscissa $\mathrm{t}[1] \mathrm{P}$ represents the predicted principal component score of the first principal component, and different shapes of the scattered points represent the different groups of the samples. E, F OPLS-DA permutation plots. The abscissa correlation coefficient represents relevance. The $Q^{2}$ and $R^{2} Y$ values reflect the model predictability and the fraction of explained variance, respectively

method and displayed in the thermodynamic diagram (Fig. 3A, B). Through further evaluation, we selected 91 and 47 differential metabolic molecules under POS and NEG. Liver metabolites mainly included esters, amino acids, carbohydrates, and lipids. Compared with the normal control group, the expression of glyceric acid, acetylcysteine, myricetin, L-arabitol, L-asparagine, tryptophan, L-phenylalaine, racemethionine, D-alanine, D-proline, $\mathrm{L}$-valine, and D-mannose were all increased in the infection group. Conversely, in the infection groups the levels of cholesterol sulfate, ribothymidine, ascorbic acid, alpha-linolenic acid, inosinic acid, adenine, thiamine, maltotetraose, creatinine, and imidazoleacetic acid were evidently decreased. Additionally, the levels of other metabolites were changed to various degrees. Faecal metabolic molecules were analysed similarly; 107 and 23 differential molecules were selected under POS and NEG (Additional file 4: Fig. S3A, B). The expression of metabolic molecules such as medicagenic acid, anandamide, piperine, N-carbamoyl putrescine, isoleucyl phenylalanine, D-aspartic acid, cytidine, and L-valine were sharply increased in the infection group. Besides, metabolic molecules such as trimethylaminoacetone, histidine, ophthalmic acid, hexadecanedioic acid, methylthioadenosine, traumatic acid, and phenylacetic acid showed a downward trend in the infection group. Coincidentally, $\mathrm{L}$-valine was upregulated in the liver and faeces in the infection group. The heat map (Fig. 3A, B) shows that the different metabolites had good classification results.

\section{Correlation analysis of different metabolites}

Complex metabolic reactions and their regulation did not occur in isolation after the mice were infected with E. granulosus. When different molecules work together, their interaction and regulation eventually lead to systematic changes in metabolomics. We calculated the correlation coefficient of the quantitative values of different metabolites and expressed it as $r$. In this study, 91 and 47 differential metabolic molecules in the liver under POS
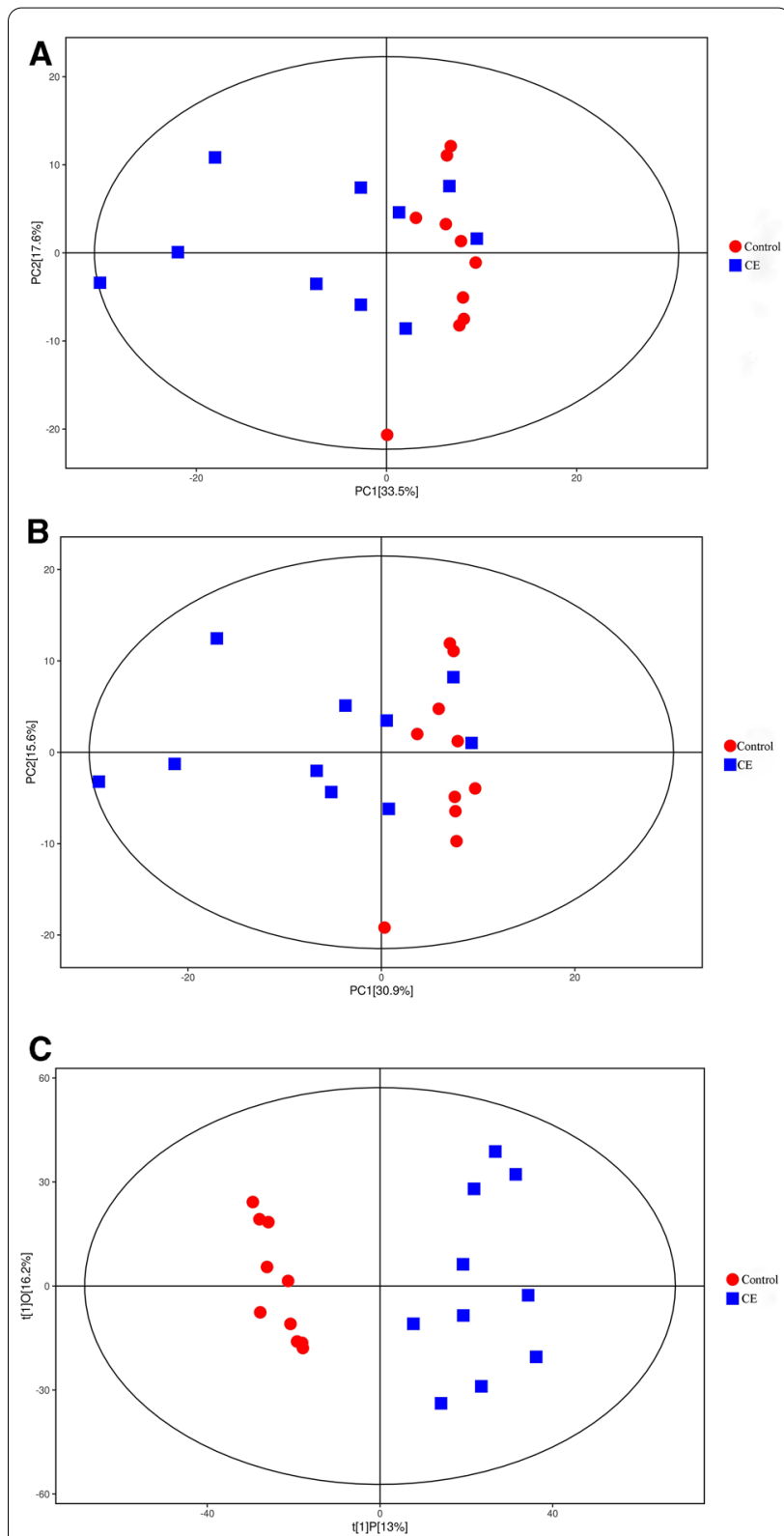

D

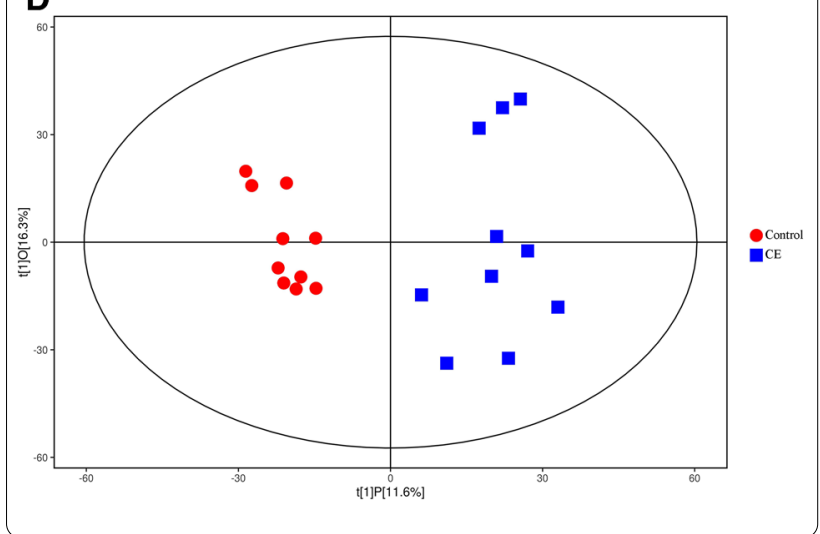



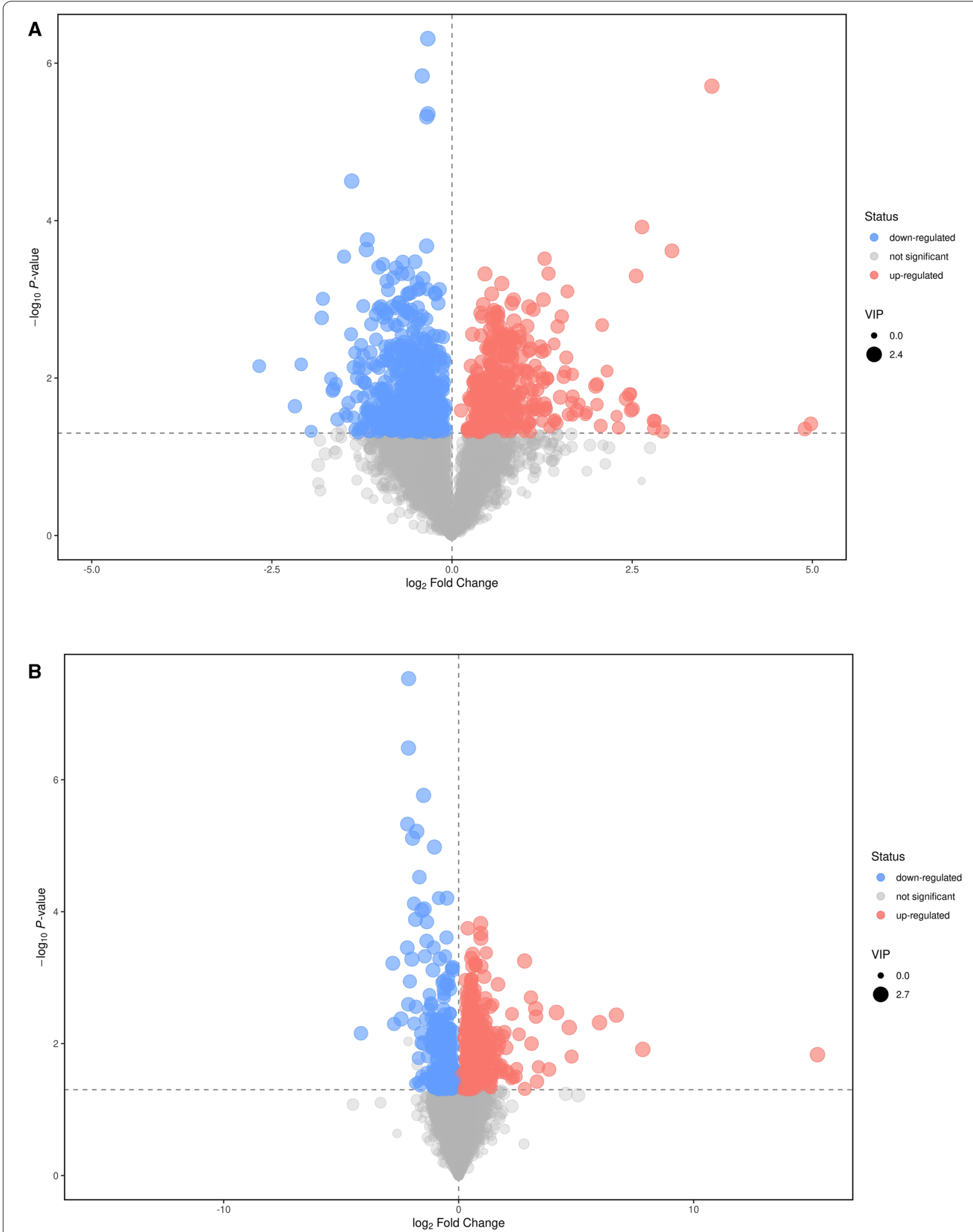

Fig. 2 Volcano plots in the liver (A, B). A, B Volcano plots. Each dot in the volcano map represents a metabolite, the abscissa shows the fold change value (take the logarithm of cardinal number 2), the ordinate represents the $p$ value of Student's $t$ test (take the negative number of base logarithm of 10), and the size of the scatter represents the VIP value of the OPLS-DA model: the larger the scatter, the greater the VIP value 
Table 1 Key differential metabolites of echinococcosis of the liver in POS

\begin{tabular}{|c|c|c|c|c|c|c|}
\hline Number & Different metabolites & $\mathrm{rt}$ & $\mathrm{mz}$ & $P$ value & Fold change & Variation treno \\
\hline 1 & DG(13:0/0:0/a-25:0) & 473.96 & 654.1 & 0.04433 & 29.7814283 & $\uparrow$ \\
\hline 2 & Deoxyviolaceinic acid & 52.113 & 358.37 & 0.00024 & 8.29206077 & $\uparrow$ \\
\hline 3 & Cytidine 2',3'-cyclic phosphate & 344.54 & 306.05 & 0.04765 & 7.59016312 & $\uparrow$ \\
\hline 4 & 6-Hydroxyprotopine & 129.38 & 370.37 & 0.01581 & 5.57671424 & $\uparrow$ \\
\hline 5 & (-)-Epiafzelechin 3-gallate & 491.7 & 427.11 & 0.0162 & 5.50331511 & $\uparrow$ \\
\hline 6 & CGP 28-392 & 132.37 & 366.34 & 0.02168 & 4.03262168 & $\uparrow$ \\
\hline 7 & $\begin{array}{l}\text { 2-(2,4-dihydroxyphenyl)-5-hydroxy-8-(hydroxymethyl)-8-methyl- } \\
\text { 4H,8H-pyrano[3,2-g]chromen-4-one }\end{array}$ & 130.6 & 369.36 & 0.01196 & 4.02562678 & $\uparrow$ \\
\hline 8 & 2-Phospho-4-(cytidine 5'-diphospho)-2-C-methyl-D-erythritol & 413.81 & 602.31 & 0.0083 & 2.96650399 & $\uparrow$ \\
\hline 9 & Bicuculline & 130.42 & 368.35 & 0.00962 & 2.92640942 & $\uparrow$ \\
\hline 10 & Methylselenopyruvate & 457.16 & 182.06 & 0.01751 & 2.83446931 & $\uparrow$ \\
\hline 11 & 2-O-(alpha-D-Mannosyl)-D-glycerate & 193.08 & 269.23 & 0.01133 & 2.40617468 & $\uparrow$ \\
\hline 12 & Glutaminylhistidine & 389.01 & 284.13 & 0.01026 & 2.39624637 & $\uparrow$ \\
\hline 13 & 4-Bromocatechol & 190.44 & 190.01 & 0.03537 & 2.37914101 & $\uparrow$ \\
\hline 14 & D-Erythrose 4-phosphate & 146.56 & 201.09 & 0.00465 & 2.36957829 & $\uparrow$ \\
\hline 15 & PC(DiMe(11,3)/DiMe(11,3)) & 448.5 & 868.17 & 0.02601 & 2.31441094 & $\uparrow$ \\
\hline 16 & Asulam & 327.76 & 372.83 & 0.00573 & 0.22063809 & $\downarrow$ \\
\hline 17 & $\operatorname{PE}(20: 3(5 Z, 8 Z, 11 Z) / 24: 1(15 Z))$ & 327.76 & 568.78 & 0.00719 & 0.28871571 & $\downarrow$ \\
\hline 18 & 2,2-Bis(4-hydroxyphenyl)hexafluoropropane & 327.75 & 960.69 & 0.02199 & 0.35378049 & $\downarrow$ \\
\hline 19 & Glucoconringiin & 327.75 & 725.8 & 0.02367 & 0.36436639 & $\downarrow$ \\
\hline 20 & Aminosalicylate sodium anhydrous & 630.94 & 279.09 & 0.00591 & 0.40899599 & $\downarrow$ \\
\hline 21 & 2'-O-Methyladenosine & 327.75 & 335.9 & 0.01821 & 0.42089842 & $\downarrow$ \\
\hline 22 & Chlorfenson & 327.76 & 668.76 & 0.00292 & 0.4254822 & $\downarrow$ \\
\hline 23 & Urate radical & 327.74 & 764.74 & 0.00112 & 0.43010083 & $\downarrow$ \\
\hline 24 & Inosinic acid & 175.43 & 120.07 & 0.01828 & 0.43609542 & $\downarrow$ \\
\hline 25 & 2-Oxoadipate & 239.73 & 240.1 & 0.0474 & 0.43874004 & $\downarrow$ \\
\hline 26 & Perfluorooctane sulfonamide & 162.66 & 796.58 & 0.0371 & 0.44433904 & $\downarrow$ \\
\hline 27 & 3'-Keto-3'-deoxy-ATP & 402.81 & 161.14 & 0.01662 & 0.46859336 & $\downarrow$ \\
\hline 28 & L-trans-4-Methyl-2-pyrrolidinecarboxylic acid & 327.72 & 962.69 & 0.00548 & 0.47930656 & $\downarrow$ \\
\hline 29 & $(2 S, 4 R, 5 S)$-Muscarine & 170.55 & 398.76 & 0.02404 & 0.48133876 & $\downarrow$ \\
\hline 30 & Cyanidin 3-(3",6"-dimalonylglucoside) & 439.59 & 178.04 & 0.02354 & 0.4816437 & $\downarrow$ \\
\hline
\end{tabular}

" $\uparrow "$ and " $\downarrow "$ mean that the compound is upregulated and downregulated

and NEG were analysed using the Pearson method. There was a clear correlation between amino acid metabolism (Fig. 4A, B, $p<0.05$ ). We also found that 107 and 23 differential molecules in the faeces observed under POS and NEG were related (Additional file 5: Fig. S4A, B, $p<0.05$ ).

\section{KEGG annotation of different metabolites}

From the KEGG pathway map, we found that the differential metabolic molecules between the infection and control groups involved multiple pathways, including energy metabolism, material transport, signal transduction, and cell cycle regulation (Fig. 5A, B). In all, 46 pathways in the liver were involved with the differential metabolic molecules. In the faeces, these differentially metabolized molecules involved 57 pathways (Additional file 6: Fig. S5A, B). For example, in the liver of the infected group, there were four differentially metabolized molecules that were enriched in purine metabolic pathways and three differentially metabolized molecules that were enriched in amino acid metabolic pathways. Similarly, in the faeces of the infected group, three differential metabolic molecules were involved in pyrimidine metabolism, and two differential molecules were involved in arginine and proline metabolism. The more the number of differential metabolic molecules involved in a certain pathway, the more notable is the difference in the metabolic pathway between the infection and control groups.

\section{Analysis of metabolic pathways of different metabolites} KEGG annotation analysis revealed all the pathways involving the differential metabolites. To determine whether these metabolic pathways were closely related to the experimental conditions, it was necessary to further analyse the metabolic pathways of the differential 


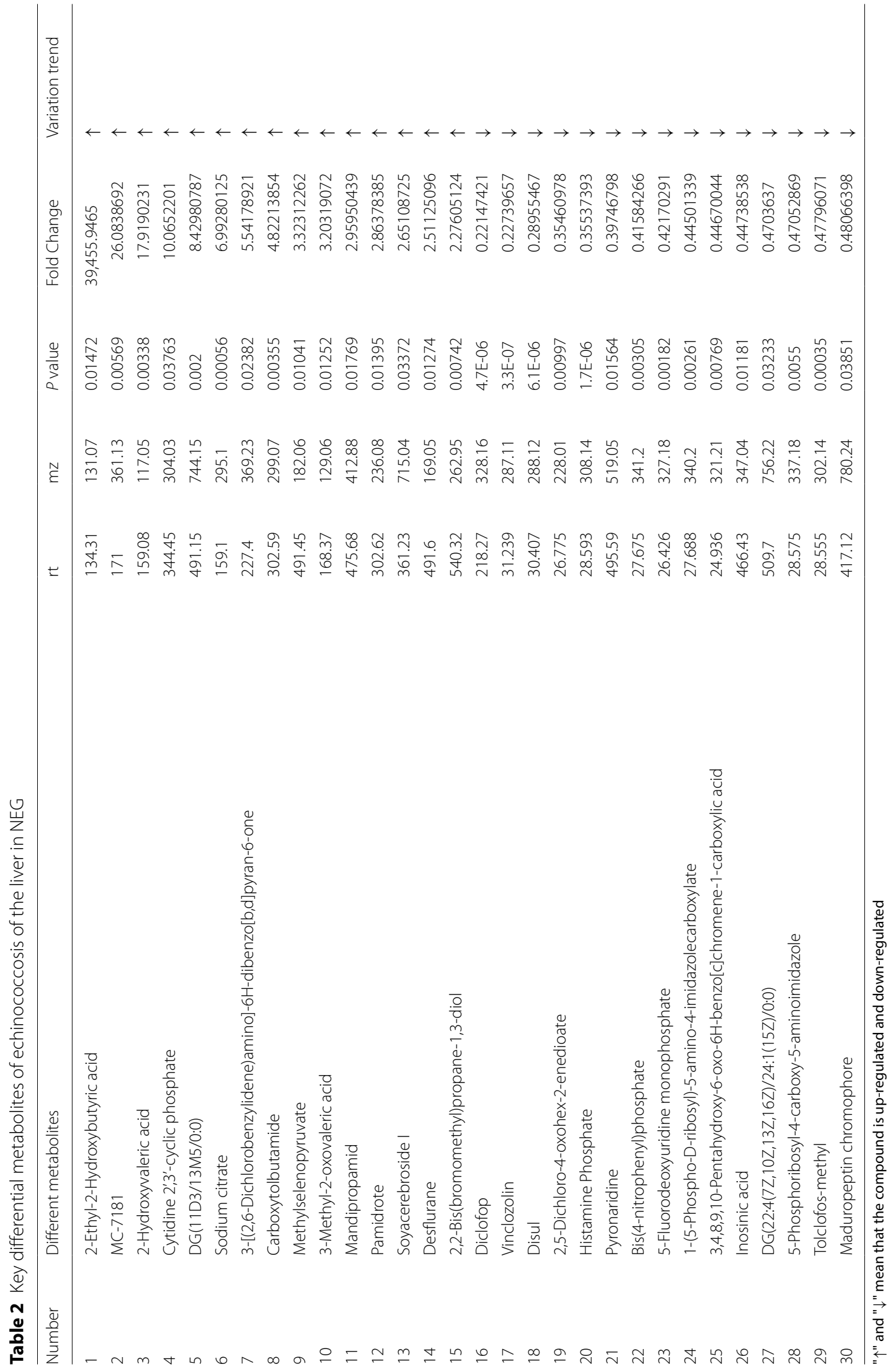




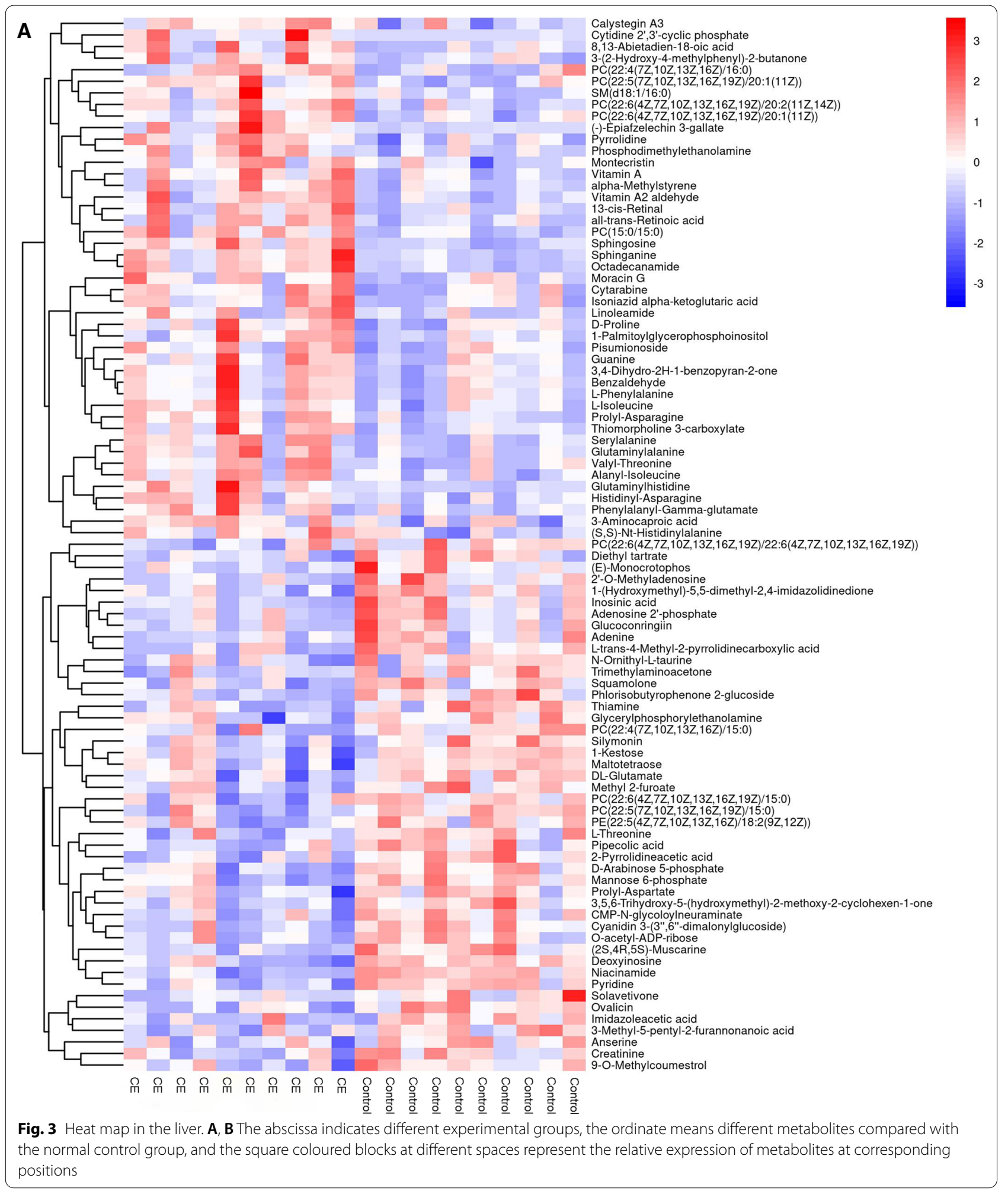

metabolites. By analysing the synthesis of the pathways (including topological analysis and enrichment analysis), we could further explore the pathways and determine the key pathways with the highest correlation with metabolic differences. The results of the metabolic pathway analysis are presented using bubble charts. In the liver, there were 


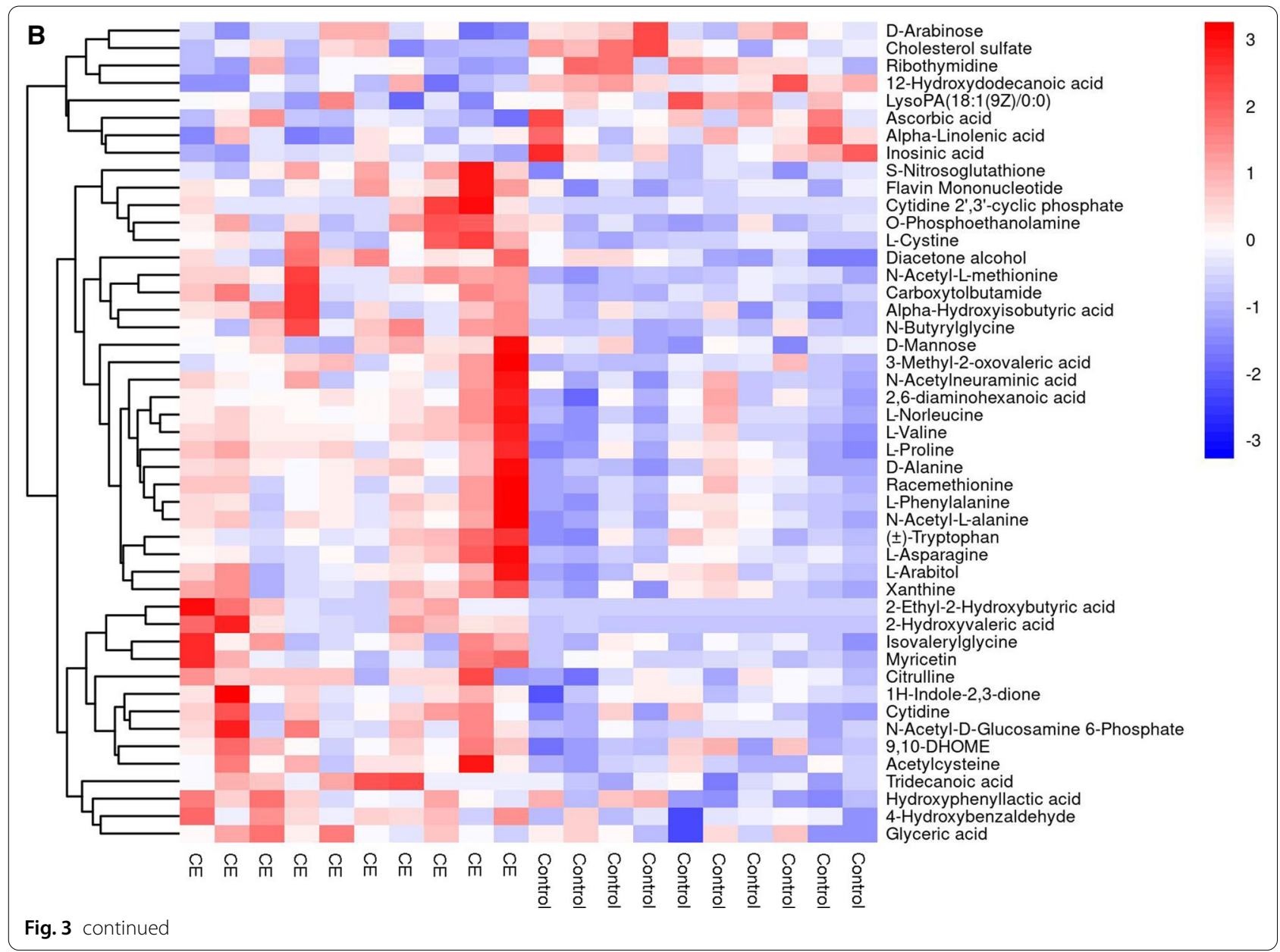

seven metabolic pathways under POS and NEG, three of which were shared. The metabolic pathways of tyrosine and tryptophan biosynthesis, phenylalanine, valine, leucine, and isoleucine biosynthesis and phenylalanine metabolism were notably associated with the occurrence and development of hydatid disease (Fig. 6A, B). However, there were 15 metabolic pathways under POS and NEG, one of which was shared (Additional file 7: Fig. S6A, B), which revealed that pantothenate and CoA biosynthesis is also significantly related to echinococcosis.

\section{Discussion}

In this study, we analysed the metabolic footprint and significant changes in metabolism, which revealed the substantial changes in the mice metabolome caused by
CE using the multivariate statistical analysis of liver and faeces. In all, 138 metabolic molecules were selected to discriminate CE mice from healthy mice. Seven metabolic pathways appeared under POS and NEG that may be related to echinococcosis. The three common metabolic pathways were tyrosine and tryptophan biosynthesis; phenylalanine, valine, leucine, and isoleucine biosynthesis; and phenylalanine metabolism, which indicated that amino acid metabolism is strongly associated with the occurrence and development of hydatid disease. These metabolic differences may provide novel insight into the biological mechanisms that occur during $\mathrm{CE}$ infection. Interestingly, 130 metabolic molecules in faeces were considered to distinguish between the infection and control groups. These molecules were enriched in

Fig. 4 Heat map of correlation analysis by group in the liver. A, B The abscissa and ordinate represent the different metabolites of the group comparison. The square coloured blocks in different positions represent the correlation coefficient between the two metabolites at corresponding positions. Red shows positive correlation, blue shows negative correlation, and the darker the colour, the stronger the correlation. Nonsignificant correlations are marked with a cross 

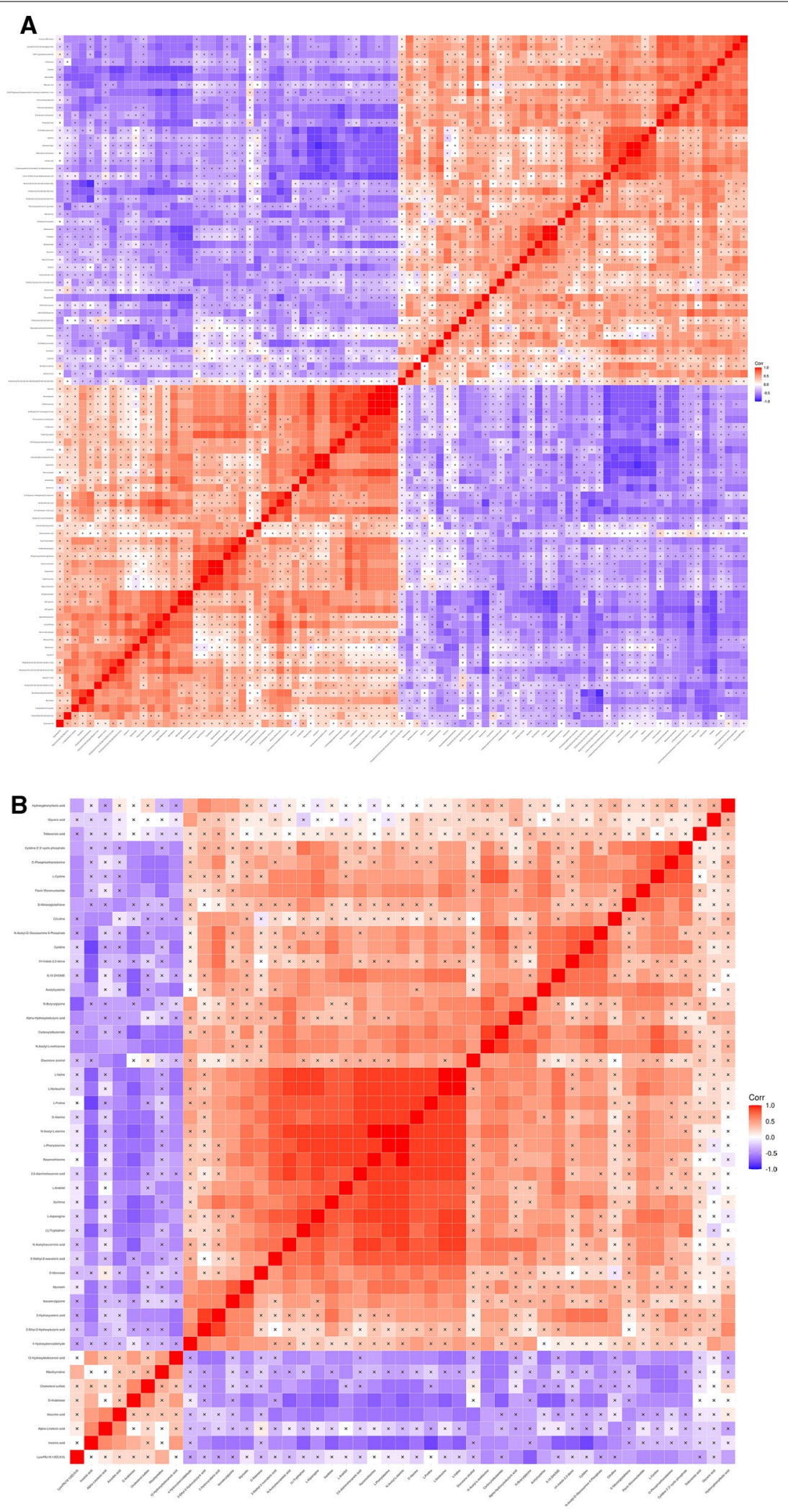


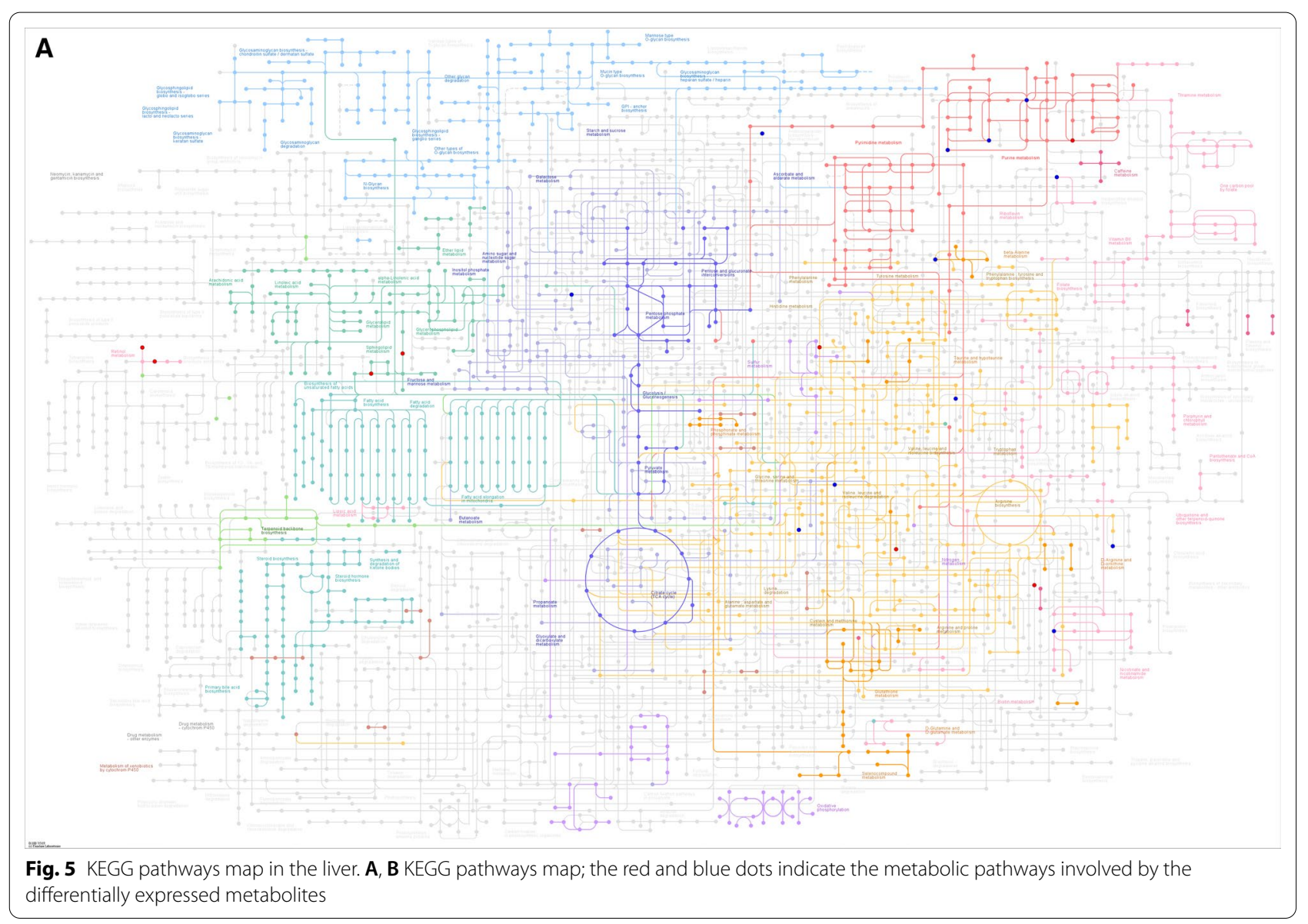

15 pathways, one of which is in common under POS and NEG. The pantothenate and CoA biosynthesis pathway indicated that the metabolism of fatty acid and pyruvate in mice with echinococcosis would be disturbed.

The main organ involved in echinococcosis is the liver. With cyst parasitization and growth, it continuously obtains nutrition from the liver. When it grows large enough, it induces change in the structure and function of the liver $[1,37]$. The results revealed that $\mathrm{CE}$ can lead to significant changes in amino acid metabolism. The circulation level of amino acids indicates the equilibrium between muscle and liver metabolism [38].

These results were confirmed by the increased phenylalanine and tyrosine levels in the infection group. Tyrosine is the primary product of phenylalanine. It has been reported that the concentrations of these two metabolites are the same. The conversion of phenylalanine to tyrosine occurs uniquely in the liver [39, 40]. The catabolism of amino acids occurs mainly in the liver [41]. Liver injury can lead to changes in amino acid metabolism, mainly manifested as a decrease in free branched-chain amino acids and an increase in free amino acids (phenylalanine, tryptophan, and tyrosine) [42]. In contrast with the normal mice, the molecular metabolites of the liver and faeces were related in the infected mice. Among the first 15 most differentially metabolized molecules, tolclofos methyl was increased in the liver and reduced in the faeces and chlorfenson was downregulated in the liver and upregulated in the faeces, while aminosalicylate sodium anhydrous and Disul were both decreased in the liver and faeces. In conclusion, the reason why amino acids (phenylalanine, tyrosine, tryptophan, valine, leucine, and isoleucine) were increased in the infection group may be related to liver dysfunction. We noticed that purine metabolism and alphalinolenic acid metabolism were abnormal in CE mice, which suggests that other pathways may participate in the metabolism after hydatid infection as well.

The limitations of this study were as follows. First, additional samples are needed to verify the current results and further improve the reliability and accuracy of the procedure. Secondly, the comprehensive application of LC-MS/ MS with GC-MS or NMR will expand the coverage of metabolomics. Moreover, a variety of omics technologies (such as genomics and proteomics) can be cross-validated and may better support the experimental results. Finally, no effective method was determined to distinguish echinococcosis from other types of liver disease in this study. 


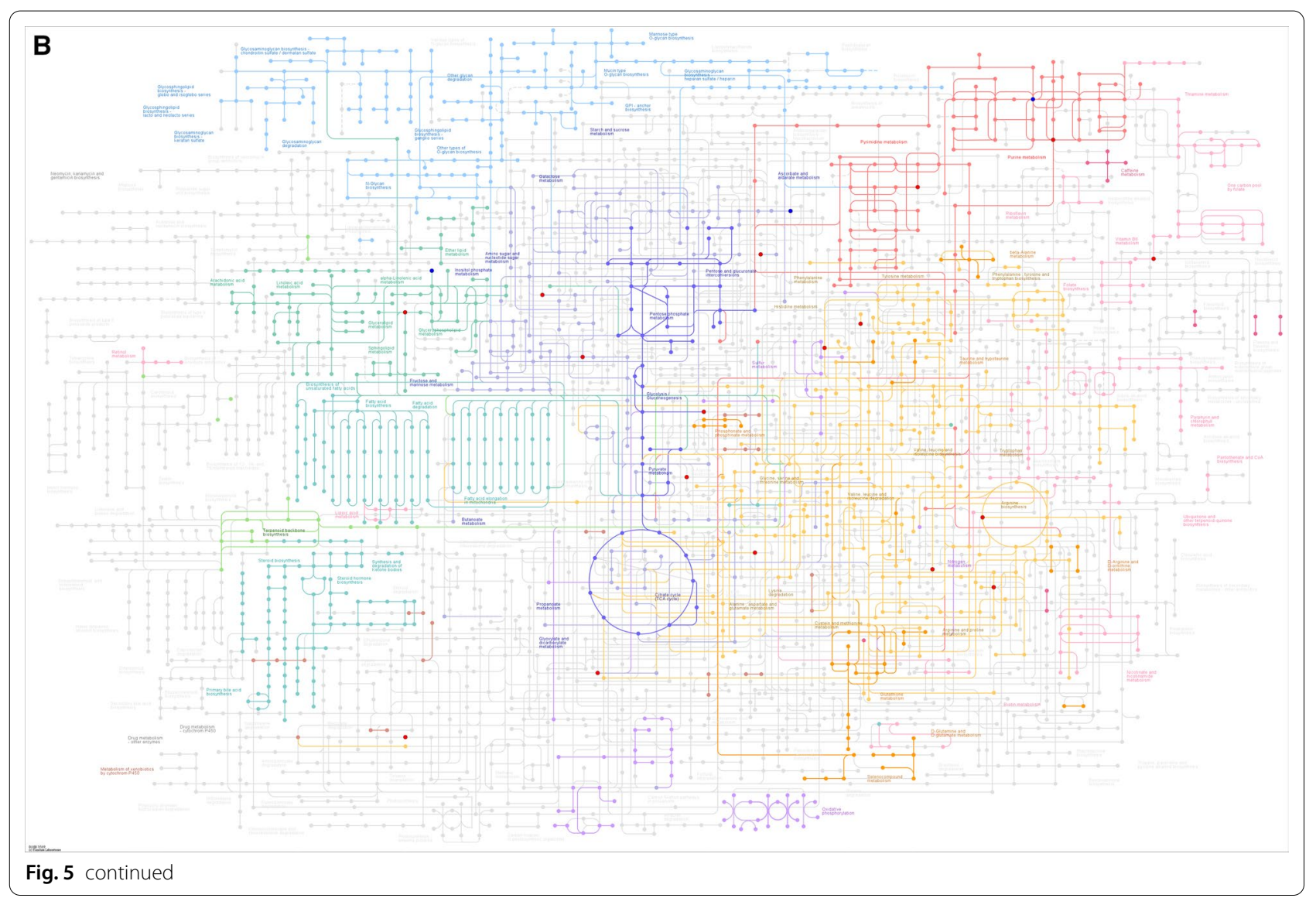

Therefore, imaging examination remains the most commonly used method for CE. If the method is developed, it is essential to preventing clinical misdiagnosis because echinococcosis is similar to other hepatic diseases. The feasibility of using the Fischer ratio and unique metabolic characteristics of echinococcosis to identify different liver diseases warrants further study. Certainly, finding a way to reduce the cost of LC-MS/MS could help to accelerate its use in clinical application.

The LC-MS/MS method is suitable for metabolomics analysis of hydatid disease due to its repeatability, multiple metabolite coverage in one measurement, and short detection duration. The systematic research of multiple metabolites of small molecules is helpful in revealing the overall metabolic changes induced by $E$. granulosus. The early diagnosis of echinococcosis may be feasible by combining both imaging techniques and metabolomics. For example, chemicalexchange-weighted magnetic resonance imaging techniques are used to yield maps weighted by the metabolites of interest [43-45]. These maps are obtained by exchanging with water and characterized by significant enhancement of the signal and the observation of small metabolic changes that cannot be observed with other imaging methods. In short, LC-MS/ MS-based metabolomics with specificity can be combined to enhance the analysis and better explain the data.

\section{Conclusion}

In our study, LC-MS/MS-based metabolomics was used to investigate metabolic diversification of $\mathrm{CE}$. It was confirmed that amino acid metabolism (phenylalanine, tyrosine, tryptophan, valine, leucine, and isoleucine) and pantothenate and CoA biosynthesis changed significantly following hydatid infection. The specific metabolic changes determined in this study may provide a new understanding of the molecular mechanism of $\mathrm{CE}$ as well

Fig. 6 Pathway analysis by group in the liver. A, B Pathway analysis by group. In the bubble plots, different bubbles represent different metabolic pathways. The abscissa and the size of bubbles indicate the influence factor of the pathway in topological analysis: the larger the size, the greater the influence factors. The ordinate and the colour of the bubble show the $p$ value of enrichment analysis (negative natural pair, i.e.-in (P)). The deeper the colour, the smaller the $p$ value, and the more significant the enrichment degree 

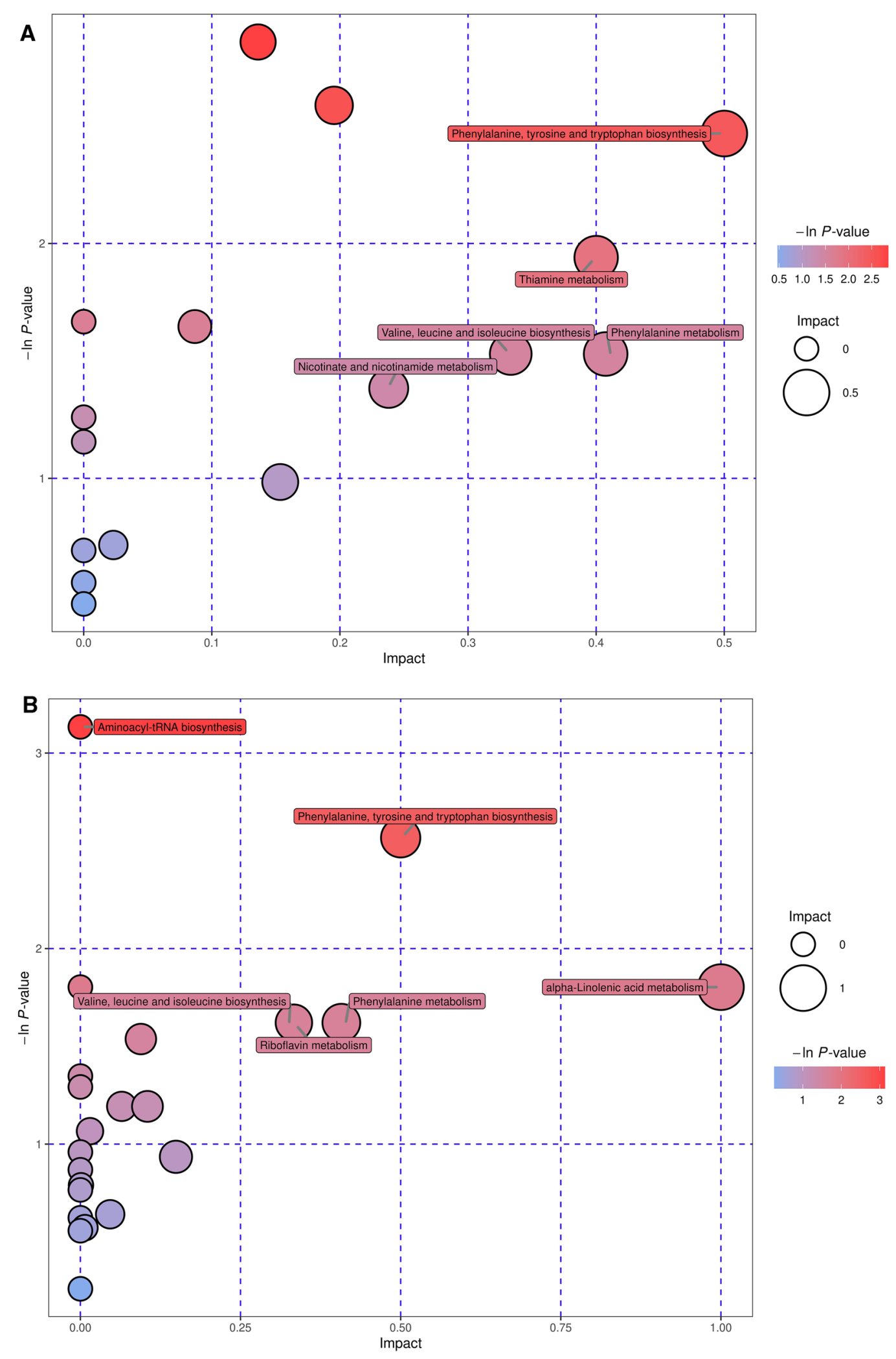
as some meaningful clues regarding the early diagnosis and therapeutic intervention in $\mathrm{CE}$.

\begin{abstract}
Abbreviations
LC-MS/MS: Liquid chromatography coupled with tandem mass spectroscopy; CE: Cystic echinococcosis; PCA: Principal component analysis; POS: Positive ion mode; NEG: Negative ion mode; IDA: Information-dependent acquisition; OPLS-DA: Orthogonal projections to latent structures-discriminant analysis.
\end{abstract}

\section{Supplementary Information}

The online version contains supplementary material available at https://doi. org/10.1186/s13071-021-04807-1.

Additional file 1: Fig. S1. PCA ( $A$ and $B), O P L S-D A(C$ and $D)$ score plots and OPLS-DA permutation plots ( $E$ and $F$ ) in faeces. A and B. PCA score plots, the abscissa PC1, and the ordinate PC2 represent the scores of the principal components ranking the first and the second, respectively, and different shapes of the scattered points represent the different groups of the samples. C and D. OPLS-DA score plots, the ordinate $t[1] \mathrm{O}$ represents the orthogonal principal component score, the abscissa t[1]P represents the predicted principal component score of the first principal component and different shapes of the scattered points represent the different groups of the samples. E and F. OPLS-DA permutation plots. The abscissa correlation coefficient represents relevance. The $Q^{2}$ and $R^{2} Y$ values reflect the model predictability and the fraction of explained variance, respectively.

Additional file 2: Fig. S2. Volcano plots in faeces (A and B). A and B. Volcano plots, each dot in the volcano map represents a metabolite, the abscissa shows the fold change value (take the logarithm of cardinal number 2), the ordinate represents the $p$ value of Student's $t$ test (take the negative number of base logarithm of 10), and the size of the scatter represents the VIP value of the OPLS-DA model: the larger the scatter, the greater the VIP value.

Additional file 3: Table S1. Key differential metabolites of echinococcosis of faeces in POS. Table S2. Key differential metabolites of echinococcosis of faeces in NEG

Additional file 4: Fig. S3. Heat map in faeces (A and B). A and B. The abscissa indicates different experimental groups, the ordinate means different metabolites compared with the normal control group, and the square coloured blocks at different spaces represent the relative expression of metabolites at corresponding positions.

Additional file 5: Fig. S4. Heat map of correlation analysis for group in faeces (A and B). A and B. The abscissa and ordinate represent the different metabolites of the group comparison. The square coloured blocks in different positions represent the correlation coefficient between the two metabolites at corresponding positions. Red shows positive correlation, blue shows negative correlation, and the darker the colour, the stronger the correlation. At the same time, the nonsignificant correlation was marked with a cross.

Additional file 6: Fig. S5. KEGG pathways map in faeces (A and B). A and B. KEGG pathways map. The red and blue dots indicate the metabolic pathways involved by the differentially expressed metabolites.

Additional file 7: Fig. S6. Pathway analysis for group in faeces ( $A$ and B). A and B. Pathway analysis for group. In the bubble plots, different bubbles represent different metabolic pathway. The abscissa and the size of the bubble indicate the influence factor of the pathway in topological analysis. The larger the size, the greater the influence factors. The ordinate and the colour of the bubble show the $p$ value of enrichment analysis (negative natural pair, i.e. - in (P)). The deeper the colour, the smaller the $p$ value, and the more significant the enrichment degree.

\section{Acknowledgements}

The authors would like to thank the Center of Scientific Technology of Ningxia Medical University for sharing the instruments.

\section{Authors' contributions}

$X C D, M X Z, H X X$, and WZ designed the experiment together. CW and SHY established the echinococcosis model in mice. TRZ and $Y Z Z$ gathered the data. XXD wrote the manuscript. MXZ and HXX provided financial support for the project. All authors read and approved the final manuscript.

\section{Funding}

This work was supported by grants from the National Natural Science Foundation of China (No. 81860366, No. 32060805), the Natural Science Foundation of Ningxia Hui Autonomous Region (2019AAC03264, 2018AAC03208), and the Foundation of Ningxia Medical University (XT2019020).

\section{Availability of data and materials}

The data of the results of this study are included in this paper and its attached files.

\section{Declarations}

\section{Ethics approval and consent to participate}

All the experiments were approved by the ethics committee of Ningxia Medical University and complied with the guidelines of all institutions and countries on experimental animals. The data obtained met the appropriate ethical requirements.

\section{Consent for publication}

Not applicable.

\section{Competing interests}

The authors declare that they have no competing interests.

\section{Author details}

${ }^{1}$ Center of Scientific Technology of Ningxia Medical University, Yinchuan 750004, Ningxia Hui Autonomous Region, People's Republic of China. ${ }^{2}$ Key Laboratory of Hydatid Disease of Ningxia Medical University, Yinchuan 750004, Ningxia Hui Autonomous Region, People's Republic of China. ${ }^{3}$ School of Basic Medical Science of Ningxia Medical University, Yinchuan 750004, Ningxia Hui Autonomous Region, People's Republic of China.

Received: 25 January 2021 Accepted: 25 May 2021

Published online: 14 June 2021

\section{References}

1. Mcmanus DP, Gray DJ, Zhang W, Yang Y. Diagnosis, treatment, and management of echinococcosis. BMJ. 2012;344:e3866.

2. Larrieu E, Gavidia CM, Lightowlers MW. Control of cystic echinococcosis: background and prospects. Zoonoses Public Health. 2019;66(8):889-99.

3. Mcmanus DP, Zhang W, Li J, Bartley PB. Echinococcosis. Lancet. 2003;362(9392):1295-304

4. Romig T. Epidemiology of echinococcosis. Langenbecks Arch Surg 2003;388(4):209-17.

5. Deplazes P, Rinaldi L, Alvarez Rojas CA, Torgerson PR, Harandi MF, Romig T, et al. Global distribution of alveolar and cystic echinococcosis. Adv Parasitol. 2017:95:315-493.

6. Larrieu E, Zanini F. Critical analysis of cystic echinococcosis control programs and praziquantel use in South America, 1974-2010. Pan Am J Public Health. 2012;31(1):81-7.

7. Craig PS, Hegglin D, Lightowlers MW, Torgerson PR, Wang Q. Echinococcosis: control and prevention. Adv Parasitol. 2017;96:55-158.

8. Tünger Ö. Epidemiology of cystic echinococcosis in the world. Turk Parazitolojii Derg. 2013;37(1):47-52.

9. Casulli A, Siles-Lucas M, Tamarozzi F. Echinococcus granulosus sensu lato. Trends Parasitol. 2019;35(8):663-4.

10. Wen H, Vuitton L, Tuxun T, Li J, Vuitton DA, Zhang W, et al. Echinococcosis: advances in the 21st Century. Clin Microbiol Rev. 2019;32(2):e18-75.

11. Kern P, Menezes Da Silva A, Akhan O, Müllhaupt B, Vizcaychipi KA, Budke $C$, et al. The echinococcoses: diagnosis, clinical management and burden of disease. Adv Parasitol. 2017;96:259-369. 
12. Romig T, Dinkel A, Mackenstedt U. The present situation of echinococcosis in Europe. Parasitol Int. 2006;55(Suppl):S187-91.

13. Frider B, Larrieu E, Odriozola M. Long-term outcome of asymptomatic liver hydatidosis. J Hepatol. 1999;30(2):228-31.

14. Agudelo Higuita NI, Brunetti E, Mccloskey C. Cystic echinococcosis. J Clin Microbiol. 2016;54(3):518-23.

15. Tao S, Qin Z, Hao W, Yongquan L, Lanhui Y, Lei Y. Usefulness of gray-scale contrast-enhanced ultrasonography (SonoVue ${ }^{\circledR}$ ) in diagnosing hepatic alveolar echinococcosis. Ultrasound Med Biol. 2011:37(7):1024-8.

16. Bujak R, Struck-Lewicka W, Markuszewski MJ, Kaliszan R. Metabolomics for laboratory diagnostics. J Pharm Biomed Anal. 2015;113:108-20.

17. Laíns I, Gantner M, Murinello S, Lasky-Su JA, Miller JW, Friedlander M, et al. Metabolomics in the study of retinal health and disease. Prog Retin Eye Res. 2019;69:57-79.

18. Jang C, Chen L, Rabinowitz JD. Metabolomics and isotope tracing. Cell. 2018;173(4):822-37.

19. Chen $X, Y u$ D. Metabolomics study of oral cancers. Metabolomics. 2019;15(2):22.

20. Filla LA, Edwards JL. Metabolomics in diabetic complications. Mol Biosyst. 2016:12(4):1090-105.

21. Ghatak A, Chaturvedi P, Weckwerth W. Metabolomics in plant stress physiology. Adv Biochem Eng/Biotechnol. 2018;164:187-236.

22. Nicholson JK, Lindon JC, Holmes E. "Metabonomics": Understanding the metabolic responses of living systems to pathophysiological stimuli via multivariate statistical analysis of biological NMR spectroscopic data. Null Xenobiotica. 1999;29(11):1181-9.

23. Uehara T, Horinouchi A, Morikawa Y, Tonomura Y, Minami K, Ono A, et al. Identification of metabolomic biomarkers for drug-induced acute kidney injury in rats. J Appl Toxicol. 2014;34(10):1087-95.

24. Wang P, Shehu Al, MaX. The opportunities of metabolomics in drug safety evaluation. Curr Pharmacol Rep. 2017:3(1):10-5.

25. Griffin JL. Twenty years of metabonomics: So what has metabonomics done for toxicology? Xenobiotica. 2020;50(1):110-4.

26. Amathieu R, Triba MN, Goossens C, Bouchemal N, Nahon P, Savarin P, et al. Nuclear magnetic resonance based metabolomics and liver diseases: recent advances and future clinical applications. World I Gastroenterol. 2016;22(1):417-26.

27. Nicholson JK, Holmes E, Kinross JM, Darzi AW, Takats Z, Lindon JC. Metabolic phenotyping in clinical and surgical environments. Nature. 2012:491(7424):384-92.

28. SenGupta A, Ghosh S, Das BK, Panda A, Tripathy R, Pied S, et al. hosts. Mol Biosyst. 2016:12(11):3324-32.

29. Wang Y, Li JV, Saric J, Keiser J, Wu J, Utzinger J, et al. Advances in metabolic profiling of experimental nematode and trematode infections. Adv Parasitol. 2010;73:373-404.

30. Fernie AR. The future of metabolic phytochemistry: Larger numbers of metabolites, higher resolution, greater understanding. Phytochemistry. 2007;68(22-24):2861-80.
31. Reaves ML, Rabinowitz JD. Metabolomics in systems microbiology. Curr Opin Biotechnol. 2011;22(1):17-25.

32. Saric J, Li JV, Wang Y, Keiser J, Veselkov K, Dirnhofer S, et al. Panorganismal metabolic response modeling of an experimental Echinostoma caproni infection in the mouse. J Proteome Res. 2009;8(8):3899-911.

33. Saric J, Li JV, Wang Y, Keiser J, Bundy JG, Holmes E, et al. Metabolic profiling of an Echinostoma caproni infection in the mouse for biomarker discovery. PLOS Negl Trop Dis. 2008;2(7):e254.

34. Saric J, Li JV, Utzinger J, Wang VL, Keiser J, Dirnhofer S, et al. Systems parasitology: effects of Fasciola hepatica on the neurochemical profile in the rat brain. Mol Syst Biol. 2010;6:396.

35. Denery JR, Nunes AAK, Hixon MS, Dickerson TJ, Janda KD. Metabolomicsbased discovery of diagnostic biomarkers for onchocerciasis. PLOS Negl Trop Dis. 2010;4(10):e834.

36. Ritler D, Rufener R, Li JV, Kämpfer U, Müller J, Bühr C, Schürch S, Lundström-Stadelmann B. In vitro metabolomic footprint of the Echinococcus multilocularis metacestode. Sci Rep. 2019;9(1):19438. https://doi.org/10. 1038/s41598-019-56073-y.

37. Eckert J, Deplazes P. Biological, epidemiological, and clinical aspects of echinococcosis, a zoonosis of increasing concern. Clin Microbiol Rev. 2004;17(1):107-35.

38. Tietge UJF, Bahr MJ, Manns MP, Böker KH. Hepatic amino-acid metabolism in liver cirrhosis and in the long-term course after liver transplantation. Transpl Int. 2003;16(1):1-8.

39. Dejong CHC, van de Poll MCG, Soeters PB, Jalan R, Olde D, steven WM. Aromatic amino acid metabolism during liver failure. J Nutr 2007:137(6 Suppl 1): 1579S-1585S

40. Abu-Omar MM, Loaiza A, Hontzeas N. Reaction mechanisms of mononuclear non-heme iron oxygenases. Chem Rev. 2005;105(6):2227-52.

41. Genchi G. An overview on D-amino acids. Amino Acids. 2017:49(9):1521-33.

42. Mcallan L, Cotter PD, Roche HM, Korpela R, Nilaweera KN. Impact of leucine on energy balance. J Physiol Biochem. 2013;69(1):155-63.

43. Cai K, Haris M, Singh A, Kogan F, Greenberg JH, Hariharan H, et al. Magnetic resonance imaging of glutamate. Nat Med. 2012;18(2):302-6.

44. Zhou J, Tryggestad E, Wen Z, Lal B, Zhou T, Grossman R, et al. Differentiation between glioma and radiation necrosis using molecular magnetic resonance imaging of endogenous proteins and peptides. Nat Med. 2011;17(1):130-4

45. van Zijl PCM, Lam WW, Xu J, Knutsson L, Stanisz GJ. Magnetization transfer contrast and chemical exchange saturation transfer MRI. Features and analysis of the field-dependent saturation spectrum. Neuroimage. 2018;168:222-41.

\section{Publisher's Note}

Springer Nature remains neutral with regard to jurisdictional claims in published maps and institutional affiliations.
Ready to submit your research? Choose BMC and benefit from:

- fast, convenient online submission

- thorough peer review by experienced researchers in your field

- rapid publication on acceptance

- support for research data, including large and complex data types

- gold Open Access which fosters wider collaboration and increased citations

- maximum visibility for your research: over 100M website views per year

At $B M C$, research is always in progress.

Learn more biomedcentral.com/submissions 